vergleichung und urgeschichte, 2. aufl. 618. E. H. Meyer, Indogerm. mythen 2,596. Mullenhoff, DA. 3,17), so bin ich geneigt, das alter desselben wie bei Griecheq Römern und Slaven, so auch bei den Germanen in die graueste vergangenheit zu verlegen. Dann gewinnt aber auch die entsprechung FjorgynnPerkunas, Perkons an gebalt und umfang (Indog. forsch. 1, 481. Delbrtick, Verwantschaftsnamen 201. Brückner, Archiv f. slav. phil. 9, 24 ff.).

HALLE A. S.

FRIEDRICH KAUFFMANN.

\title{
DER SIEGFRIEDMYTHUS.
}

Die Sturlaugssaga starfsama FAS. 3, 633 ff. enthält folgende episode:

König Sturlaugr hat am julabend das gelubde getan, den ursprung des úrarhorn in erfahrung zu bringen, das er $\mathbf{8 . 6 3 0}$ nach einem gefahrvollen abenteuer in Bjarmaland dem könig Haraldr gebracht hat. Er erhält von der alten Véfreyja, der pflegemutter (fostra) seiner gemablin Ása, den rat, welchen er im folgenden ausfthrt. Sturlaugr fordert nämlich den Frosti, mit welchem er s. 615 blutsbruderschaft geschlossen bat, auf, fur ihn um die Mjoll, die tochter des könig Snær in Finnmarken, zu werben. Er gibt ihm einen runstab (kefli) mit, welchen Frosti der Mjoll in den schooss werfen soll. Frosti kommt zu könig Snær und nennt sich Gestr. In der nähe der königlichen balle befindet sich ein gemach, von hohen holzwänden (skiðgarðar) umgeben, die so hoch sind, dass nur ein vogel im fluge hinuber kommen kann. Darin haust die königstochter Mjoll. Frosti hält sich immer in der nähe der skiðठgarðar auf, um die königstochter zu sehen, aber den ganzen winter tuber gelingt ihm das nicht. Da findet er eines tages die skiðgarðar und das gemach der königstochter offen, er tritt ein und sieht eine frau auf einem stuble sitzen, die mit einem goldkamme ibr haar, schön wie seide, kămmt. Niemals glaubt 
er ein schöneres weib gesehen zu haben. Da wirft er ihr den runstab, welchen er vom könig erhalten hat, in den schooss; sie liest und lächelt dem Frosti freundlich zu. Frosti oder Gestr, wie er sich nennt, kann nicht schlafen noch essen vor sorge um den zweck seiner reise. In der nacht wirft ihm die königstochter einen goldring zu, er geht hinaus und trifft dort Mjoll. Sie fragt ihn, ob das wahr sei, was auf dem runstab stehe, er bejaht es und sie erklärt sich bereit, die geliebte (friðla) des königs zu werden, den sie mehr schätze als alle ubrigen männer. Mjoll entflieht mit Frosti vom hof ihres vaters mit zauberhafter schnelligkeit, denn sie hat einen zaubergurtel, an welchen sich Frosti anhält, um schneller vorwärts zu kommen. Als sie in Schweden sind, und Frosti dem Sturlaugr mitteilt, dass er Mjoll mitgebracht habe, sagt Sturlaugr befriedigt: jetzt ist der fuchs aus seiner böhle gegangen; du sollst sie nun beiraten und meine besten kleider anziehen, dann wird sie glauben, dass ich es sei, denn wir sehen uns beide sehr ähnlich. Dann verlangt Sturlaugr von Frosti, dass er die Mjoll, wenn er mit ibr im bett liege, fragen solle, wie das úrarhorn entstanden sei, denn sie allein wisse das. Er selbst, Sturlaugr, werde ungesehen ihr gespräch belauschen. Frosti tut, wie ihm befohlen wurde. Die braut ist sehr glueklich, sie schläft in der nacht mit Frosti, welchen sie fur den Sturlaugr hält und mit Sturlaugr minn anspricht. Frosti fragt sie nach dem ursprung des úrarhorn, denn er habe gelobt, denselben in erfahrung zu bringen. Sie gibt ihm den gewlunschten bescheid und Sturlaugr bört ibr gespräch. Als Mjoll ibre erzählung geendet hat, geht Sturlaugr weg und lässt das gemach, in welchem Mjoll und Frosti liegen, in brand stecken. So kommen sie beide ums leben. Sturlaugr hat das auf den rat der Véfreyja getan, denn Mjoll war sehr zauberkundig und hätte dem Sturlaugr und der Véfreyja geschadet, wenn sie das vorausgesehen hătte.

Die Sturlaugssaga erzählt also von einem mädchen, das hinter hoben mauern wohnt, thber welche nur ein vogel gelangen kann. Ein könig wirbt durch einen abgesanten um das mädchen, aber er täuscht es und liefert es dem boten aus, der ihm sehr ähnlich sieht und dem er ausserdem seine kleider zu diesem zwecke gibt; das mädchen schläft mit dem boten in der meinung, dass er der könig sei. 
Setzt man für Mjoll, Sturlaugr, Frosti die namen Brynhildr, Gunnarr, Siguror ein, so erhält man die Siegfriedsage mit allen hauptzügen, mit dem Vafrlogi, dem gestaltentausch, dem beilager, so dass eigentlich nur der schluss verschieden ist. Wenn das mädchen von bohen skiðgarðar umgeben ist, so ist daran zu erinnern, dass auch in der Volsungasaga Brynhildr auf einem hohen turm haust. Ein zufall ist hier völlig ausgeschlossen, denn unmöglich können so eigenartige motive an zwei punkten unabhängig neben einander erscheinen, und neben diesen ubereinstimmungen kann die tatsächlich vorhandene verschiedenheit, dass in der Siegfriedsage Siguror, in der Sturlaugssaga aber Sturlaugr, welcher dem könig Gunnarr entspricht, das mädchen ausliefert, gar nicht in betracht kommen. Fraglich ist nur, wie man sich das verhältnis der beiden fassungen $\mathrm{zu}$ denken hat.

Die Sturlaugssaga macht gewiss nicht den eindruek eines hohen alters. Fremder einfluss ist ganz deutlich in den namen Aldeigjuborg (Ladoga) s. 640 und Heinrekr, wie der sohn des Sturlaugr s. 647 heisst. Auch die vorstellung vom úrarhorn kann nicht skandinavisch sein, da der ur in Skandinavien im mittelalter nicht mehr vorkam. Sie muss aus Deutschland oder Russland stammen, wahrscheinlich aus letzterem, denn das tier wird in Bjarmaland an der Vína von könig Haraldr erlegt, s. 637, und auch der tempel, in welchem das horn aufbewahrt wird, ist in Bjarmaland, s. $626 \mathrm{ff}$.

Man denkt zunächst an die möglichkeit, dass die episode der Sturlaugssaga die Siegfriedsage voraussetzt. Aber dann musste eine mythologische rluckbildung stattgefunden haben, es mussten die namen der Siegfriedsage durch die mythischen, Mjoll, Snær, Frosti, ersetzt worden sein. Dabei fällt aber sofort auch auf, dass in den offenbar verwanten Fjolsvinnsmál der mann, welcher zu Menglo $\delta$ dringt, also dem Frosti entspricht, sich Vindkaldr nennt, und angibt, der sohn eines Vảrkaldr zu sein. Es stehen ferner den früher erwähnten fremden zügen der saga auch echt mythische gegenuber. So heisst es s. 631, dass zur zeit des Sturlaugr in Schweden Ingifreyr könig war, und vor allem ist die Véfreyja mythisch.

Sie ist die pflegemutter der Ása, der gemahlin des Sturlaugr. S. 594 wird sie als ein altes weiblein geschildert, mit 
vor alter roten augen, das flachs spinnend in einem gemache sitzt, von welchem sie jeden ankömmling sehen kann. Wer denkt bier nicht an die alte spinnerin im märchen vom dornröschen? Ja selbst der kleine zug, dass die alte darüber erbost ist, dass man sie nicht eingeladen hat, findet sich bei Véfreyja, allerdings in einem ganz andern zusammenhange. S. 605 beklagt sich Véfreyja dem Sturlaugr gegentiber, dass sie nicht zur hocbzeit ihrer pflegetochter Ása geladen wurde. Der fóstri der Véfreyja ist Svipuðr, d. i. offenbar Óðinn, der ja sonst als Svipall oder Svipdagr blindi erscheint, 8. 0. s. 93. Wenn im märchen die alte spinnerin das mädchen mit der spindel, in der Siegfriedsage aber Óðinn die Sigrdrífa mit dem schlafdorn sticht, so ergibt sich leicht die sage, wie sie in der Sturlaugssaga vorliegt, wo Véfreyja und Svipuðr neben einander erscheinen, als die vom märchen wie von der Siegfriedsage vorausgesetzte, natürlich nicht unmittelbare quelle. Von dem schlaf des mädchens weiss allerdings die Sturlaugssaga nichts. Véfreyja und Svipurr sind der Mjoll nur feindlich gesinnt, aber es muss jetzt dieses motiv auch fur die ursprungliche fassung der geschichte von Sturlaugr und Mjoll angenommen werden, wie der nachstehende stammbaum lehrt:

Sturlaugssaga
Vefreyja, Svipuor (schlaf)
$\begin{gathered}\text { märchen } \\ \text { schlimine alte, schluf }\end{gathered}$

Wenn Sturlaugr, der mit der Véfreyja befreundet ist, der die pflegetochter der Véfreyja zur gemahlin bat und ein zeitgenosse des Ingifreyr ist, durch einen boten um die von hohen skiðgarðar umgebene Mjoll wirbt, so erinnert das an den mythus von Freyr; Gerðr und Skśrnir. Sn. E. 1,120 wohnt Gerðr in einem mikit his, Freyr kann vor liebessehnsucht nicht schlafen und trinken. FAS. 3,635 heisst es von Frosti, nachdem er Mjoll getroffen hat: hverki mátti njóta svefns né matar fyrir áhyggju peirri, er hann haf $\partial i \dot{a}$ ferd sinni. Man beachte ferner die beschreibung der Mjoll 3,634: hann (Frosti) gengr inn, ok sér at par sitr kona á stóli ok kembir sér með gullkambi, hárit lá á dýnunni hjá henni, fagurt sem silki. Hann sér nú yfirlit hennar, ok póttist hann eigi hafa séd fegri konu en bessa. 
Aber Mjogll (schnee), die tochter des Finnenkönigs Snær (schnee), die von Sturlaugr verschmäht wird, erinnert auch an Skaði, die in der Ynglingasaga als Drifa (schneesturm), Skjálf (beben vor kälte) mit ihrem vater Snjár (schnee) oder Frosti (frost) erscheint und welche von Njoror verlassen wird.

Sn. E. 1, $214 \mathrm{ff}$. kommt Skaði in helm und brunne nach Ásgaror, um busse für die ermordung ihres vaters $D_{j a z i}$ von den asen zu fordern. Sie verlangt, dass sie sich einen mann aus den göttern wăhlen dürfe, und das gewähren ihr die asen, nur muss sie die wabl at fótum, nach den fussen, treffen und sonst nichts von den göttern sehen. Sie wählt den Njoror mit den worten fátt mun ljótt á Baldri, glaubt also den Baldr zu wählen. Skaði macht aber noch eine zweite bedingung, nämlich dass die götter sie zum lachen bringen sollen; sie glaubt, es werde ihnen das nicht gelingen, aber Loki bringt es zu stande durch seinen unflătigen scherz. Wenn die götter die Skað̧i nur die füsse sehen lassen und Skaði hierauf den Njọror wählt, aber den Baldr wählen will, so kann das offenbar nur den sinn haben, dass die gōtter die Skað̃i täuschen wollen: sie wollen nicht, dass sie den besten von ibnen, den Baldr, nimmt, was offenbar geschehen wäre, wenn sie sich ihr ganz gezeigt hätten, und diese list gelingt. Dass Skaði als entschädigung fur die ermordung ihres vaters verlangt, dass sie sich einen mann aus den asen wählen durfe, ist ganz verständlich, aber sinnlos ist ihre zweite forderung, dass sie zum lachen gebracht werden soll. Man hat den eindruck, es nur noch mit den trummern von einem mythus $z u$ tun zu baben. Aber es făllt auf, dass hier zwei zuge erscheinen, welche sich auch in der geschichte von Sturlaugr und Mjoll finden, denn man hat schon längst mit der Skaði, welche Loki zum lachen bringt, die schlafende valkyre verglichen, welche Sigurðr erweckt, und wenn Skað̋i getäuscht wird und den Njọrðr erhălt, während sie den Baldr will, so erinnert das an die Mjoll, welche Frosti für den Sturlaugr hält.

Nimmt man nun als ursprungliche gestalt des Skaðimythus an, dass Njortr um die Skað̃i (Mjoll), welche in zauberhaftem schlafe liegt, durch seinen boten, Frosti, der sie erweckt, wirbt, dass er sie dann verlässt und dem Frosti, der ihm sehr ähnlich sieht, ausliefert, also im wesentlichen die geschichte von Mjoll 
und Sturlaugr oder die Siegfriedsage, so kommt mit einem male ein zusammenhang in die erzählung der Sn. E. Denn dann brauchte man nur die änderung vorzunehmen, dass Skari sich ihren mann selbst wählt, so konnte sie nicht mehr schlafen: das motiv vom zauberhaften schlaf musste entweder gestrichen oder geändert werden: man wählte das letztere und setzte daftir das bekannte novellenmotiv vom mädchen, welches nicht lachen kann (vgl. die Cunneware), ein, welches man, so gut es gieng, in einen zusammenhang zu bringen suchte. Es wurde ferner das motiv von der täuschung der Skaði und der verwechslung vorgerlickt und in die zeit der gattenwahl verlegt; da nun Skaði den Njorðr heiratet, so musste sie den Njorðr wählen; derjenige, welchen sie wählen will, musste dann ein besserer als der Njorðr, der Baldr, sein. Ich finde hierin keine geringe bestätigung für die richtigkeit meiner combination, dass sich jetzt die mythentrummer, welche uns Snorri therliefert hat, zu einem ganzen zusammenftigen. Nach Ynglingasaga c. 9 beiratete Skað̋i später den Óðinn, welchem sie den Finnen Sæmingr gebar. Die Mjoll leiratet den Frosti; ein sohn der beiden wird allerdings nicht genannt, aber Skaði und Frosti sind gewiss ein passendes elternpaar für den Lappen Sæmingr. Es wird also in der fassung des Skarimythus, welche Snorri kannte, nur das motiv von der verwechslung vorgertickt worden sein, die zweite heirat aber ihren alten platz behalten haben. Die echtheit des mythus von Sturlaugr und Mjoll darf also jetzt fur erwiesen gelten. Sturlaugr ist Njgror oder Freyr und Mjoll ist die Skaði. Das verhältnis zum Siegfriedmythus kann nur so gedacht werden, dass beide auf eine gemeinschaftliche quelle zurückgehen. Es ist das dieselbe, welche wir oben für die erzählung Snorris angenommen haben. In ihr fand sich der zug, dass Njorðr die Skaði heiratet und verlässt, worauf erst die auslieferung an Frosti folgte, denn derselbe erscheint sowol im Skaðimythus, als auch in der Siegfriedsage. Es ist ferner deutlich, dass die Siegfriedsage insofern nicht das ursprügliche bewahrt hat, als hier Siegfried, der bote, die ralkyre verlässt, also dem Njọrðr entspricht, während der könig den Frosti vertritt. Die abweichung erklärt sich leicht aus der verbindung des Siegfriedmythus mit der historischen Burgundensage, denn wenn könig Gunnarr an die stelle des Frosti trat, 
konnte er unmöglich in der untergeordneten stellung eines boten belassen werden.

Der mythus von Freyr und Gerðr hat das alte verbältnis vom gott zu seinem diener bewahrt, aber die sage hat hier einen guten ausgang ohne conflict erhalten. Man hat ferner auch das paar Mjoll und Sturlaugr mit dem paare Véfreyja (Menglo ) und Svipuor (Svipdagr) vertauscht, den boten Frosti zunächst als Vindkaldr beibehalten, später den namen Vindkaldr zu einem pseudonym des Svipdagr gemacht, und der sage denselben glücklichen ausgang wie im Gerormythus gegeben, und auch das märchen vom Dornröschen geht auf dieselbe quelle zurtick, wie die Siegfriedsage, und ist nicht erst aus derselben abgeleitet, da hier die Véfrey,ja das mädchen in schlaf versenkt, in der Siegfriedsage aber Öinn. Wir werden jetzt auch zu einer andern auffassung des namens Sigrdrífa gefuhrt. Es ist der zweite compositionsbestandteil -drifa zu betonen, denn Sigrdrifa ist mit der Drifa (Skaði) der Ynglingasaga identisch, und der name Sigrdrifa verhält sich zu Drifa, wie sich etwa Herebeald zu Baldr verhält, s. o. s. 85.

Von der Drífa erzählt Ynglingasaga c. 16, dass sie den Vanlandi, der sie verlassen hat, aus rache fur seine untreue durch die seiðkona Huldr töten lässt. Aehnlich ist die erzählung Ynglingasaga c. 22 von der Skjálf, und auch Gísl und Ọndurr rächen ihre mutter an Vísburr c. 17. Man darf wol jetzt damit die rache der Brünhild vergleichen, so dass die ganze Siegfriedsage ihre erklärung in dem Skaðimythus gefunden hat. In der geschichte von Sturlaugr und Mjoll hat der schluss eine andere wendung bekommen nach art der hexen- und trollkonurgeschichten. Die Mjoll wird mit Frosti verbrannt, aber die trollkona-natur und gefährlichkeit der Mjoll wird hervorgehoben. Ich nehme an, dass es eine fassung des Skarimythus gegeben habe, wo Drifa oder Mjoll ihren zweiten gemahl Frosti gegen Njoror aufgereizt und so dessen untergang berbeigefuhrt hat. Das steht nicht zu weit ab von Ynglingasaga e. 17, wo Gísl (skistock) und Ọdurr (schneeschub) gegen ihren vater Vísburr auftreten, und auch von einer zweiten heirat des Vísburr wird hier berichtet.

Ich habe oben in meinem aufsatz 'Zur Ynglingasaga' s. $78 \mathrm{ff}$. zu zeigen versucht, dass der schatzmythus der Nibelungen- 
sage identisch ist mit dem Djazimythus. Die brlder Scbilbunc

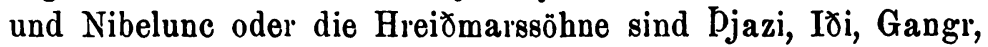
die söbne des Auðvaldi. Somit bat nicht nur die Siegfriedsage, sondern die ganze Nibelungensage bis Siegfrieds tod ihre entsprechung in der geschichte des geschlechts der Skazi. Man mag noch hinzu halten die geschichte von Handvanus (Andvari), der bei Saxo dem Hadingus (Njor rr) seinen schatz ausliefern muss, s. 0. s. 81, und die drachenkämpfe, welche Saxo bei Frotho I. s. 61 ff. und bei Fridlevus 271 erzäblt, welche beide Freyr sind. Es muss hier ferner daran erinnert werden, dass im eingang der Volsungasaga bei Sigi, dem ahnherrn des Volsungengeschlechts, der Skaðimythus erscheint. Sigi, der sohn des Óð̇inn, welcher den Breði, den diener des Skaði, tötet und dafur verbannt wird, ist aller wahrscheinlichkeit nach Njorðr, vgl. oben s. 79 .

Wir sind in dieser untersuchung so weit gelangt, als wir es nur wünschen können, nämlich bis zu einem naturmythus mit ganz durchsichtigen namen. Wenn Njorðr die Skaðri heiratet, sie verlässt und dem Frosti ausliefert, der fur sie ein passenderer mann ist als Njorðr, so ist der gegensatz von sommerlicher und winterlicher natur ganz deutlich. Bei den einzelheiten wird man freilich nur raten können. Es vergleicht sich auch die vortbergehende ehe des Njoryr mit der Finnin Skaði der vorubergehenden ehe der Frigg mit dem Finnen Ullr: wir haben es hier offenbar mit parallelmythen zu tun.

Es ist aus dem vorhergehenden klar geworden, dass Sigrdrifa und Brynhildr eine und dieselhe mythische gestalt, die Skaði, sind. Man wird also jetzt nicht mehr an Heinzels ausführungen WSB. 1885, s. 695 ff. festhalten können, wonach die valkyren Sigrdrifa und Brynhildr ursprtinglich als verschiedene wesen aufgefasst wurden. Heinzel hat gewiss mit recht darauf aufmerksam gemacht, dass es nach der vorstellung des dichters der Grípisspá zwei valkyren, Sigrdrífa und Brynhildr, gab. Aber wenn Heinzel meint, es sei wol verständlich, dass, sobald die sage zu biographischer behandlung vorschritt, sich eine ästhetische veranlassung ergab, aus den zwei valkyren eine zu machen, der umgekehrte weg aber sei unverständlich, so ist dagegen einzuwenden, dass sich die spätere doppelheit ganz wol erklärt aus dem streben nach häufung der motive, welches in 
der sagaliteratur so oft dazu gefthrt hat, dass eine holmganggeschichte oder eine gefährliche werbung mit nahezu den gleichen motiven der andern folgt.

Dagegen gewinnt die zweite hypothese Heinzels, dass die verbindung der historischen Burgundensage mit dem Siegfriedmythus im norden vor sich gegangen ist, jetzt sehr an wahrscheinlichkeit. Da in Deutschland ein weiblicher Njorðr bezeugt ist, so ist die möglichkeit nicht ausgeschlossen, dass es auch einen deutschen Skaðimythus gegeben habe. Aber auch dann könnte derselbe nur fur den norden Deutschlands in anspruch genommen werden. Wie uns der Skaðimythus vorliegt, setzt er notwendig skandinavische verhältnisse voraus, den gegensatz von Finnen und Germanen, und vor allem hochland. Die hohen mauern, hinter welchen Mjoll (schnee) wohnt, sind doch wol die hohen berge. Dafur dass der Nibelungenmythus aus Skandinavien nach Deutschland gekommen ist, sprechen auch ziemlich bestimmt die von Heinzel a. a. 0. 712 hervorgehobenen tatsachen, die localisierung der Brtinhild auf Island, der Nibelungen in oder bei Norwegen. Dagegen können zugge wie der, dass Siguror bei der schwertprobe das schwert in den Rhein hält, oder dass er inn suðrœni genannt wird, die deutsche heimat des Nibelungenmythus nicht beweisen, denn notwendig musste nach der verbindung mit der Burgundensage auch der mythus am Rhein localisiert werden. Wir werden uns also wol dazu verstehen mussen, die heimat der Bribnhild und unseres Dornröschens nicht in Deutschland, sondern in Skandinavien zu suchen.

WIEN.

FERD. DETTER.

\section{HÁRR.}

Ich habe in meinem aufsatz 'Zur Ynglingasaga' einige sprachliche mythenerklärungen versucht, d. h. angenommen, dass ein mythus erfunden werden konnte zur erklärung eines poetischen ausdrucks, dessen urspringliche bedeutung nicht mehr verstanden wurde. Man wird wol zugeben milssen, dass mit solchen möglichkeiten bei der nord. poesie, die so sehr mit 